\title{
Diagnóstico de sobrepeso e obesidade em escolares: utilização do índice de massa corporal segundo padrão internacional
}

\author{
Diagnosis of overweight and obesity in schoolchildren: \\ utilization of the body mass index international standard
}

\author{
Rodolfo Giugliano1, Ana L. P. Melo
}

\section{Resumo}

Objetivo: Avaliar a concordância entre o índice de massa corporal segundo padrão internacional e indicadores de adiposidade no diagnóstico de sobrepeso e obesidade em escolares.

Métodos: Avaliação de peso e estatura de 528 escolares, entre 6 e 10 anos, de ambos os sexos, seguida da classificação das crianças em eutróficas, com baixo peso, sobrepeso ou obesidade segundo índice de massa corporal por idade. A adiposidade foi estimada a partir da somatória das medidas das dobras cutâneas tricipital e subescapular, tricipital e da panturrilha medial e pelas circunferências da cintura e quadril.

Resultados: A prevalência de sobrepeso e obesidade foi de $21,2 \%$ nas meninas e $18,8 \%$ nos meninos. A porcentagem de gordura corporal média nas crianças normais $(17,7 \%)$ foi significativamente diferente da observada nas crianças com sobrepeso $(27,0 \%)$ e obesidade $(29,4 \%)$ $(p<0,02)$. As circunferências médias da cintura e quadril dos meninos e meninas eutróficos $(56,9 \mathrm{~cm}$ e $67,7 \mathrm{~cm})$ diferiu significativamente daqueles com sobrepeso $(65,9 \mathrm{~cm}$ e $77 \mathrm{~cm})(p<0,01)$. Não houve diferença na razão cintura-quadril entre os grupos. A porcentagem de gordura corporal, a circunferência da cintura e a circunferência do quadril correlacionaram-se significativamente com o índice de massa corporal $(p<0,01)$.

Conclusão: O uso do índice de massa corporal por idade, baseado em padrão internacional, mostrou-se adequado para o diagnóstico de sobrepeso e obesidade na faixa etária estudada, apresentando boa concordância com a adiposidade.

J Pediatr (Rio J). 2004;80(2):129-34: IMC, pregas cutâneas, circunferência da cintura, circunferência do quadril, razão cinturaquadril.

\section{Introdução}

A obesidade vem aumentando de forma alarmante, sendo considerada uma verdadeira epidemia mundial, atingindo todas as faixas etárias, especialmente as crianças ${ }^{1}$. Nos EUA, comparando-se os inquéritos nacionais de 1965 e

1. Doutor. Pediatra, professor titular da Universidade Católica de Brasília (UCB).

2. Mestre em Educação Física.

Artigo submetido em 14.10.03, aceito em 21.01.04.

\section{Abstract}

Objective: To evaluate the agreement between the body mass index international standard and body fat for the diagnosis of overweight and obesity in schoolchildren.

Methods: 528 schoolchildren between 6 and 10 years of age, of both sexes, had their weight and height measured. They were then classified as normal, underweight, overweight or obese using the body fat index for age. Body fat was estimated based on the sum of triceps and subscapular, triceps and calf skinfold measurements and waist and hip circumference.

Results: Overweight and obesity were found in $21.2 \%$ of the girls and $18.8 \%$ of the boys. The average percentage of body fat in the normal children $(17.7 \%$ ) was statistically different from that of overweight $(27 \%)$ and obese $(29.4 \%)$ children $(p<0.02)$. The average waist and hip circumference also differed significantly between normal boys and girls $(56.9 \mathrm{~cm}$ and $67.7 \mathrm{~cm})$ and overweight children $(65.9 \mathrm{~cm}$ and 77 $\mathrm{cm})(\mathrm{p}<0.01)$. There was no significant difference between groups in terms of average waist to hip ratio. The percentage of body fat, waist and hip circumference were significantly correlated with body mass index $(p<0.01)$.

Conclusion: Body mass index for age was an adequate indicator of overweight and obesity in group studied, with a good agreement with body fat.

J Pediatr (Rio J). 2004;80(2):129-34: BMI, skinfold measurements, waist circumference, hip circumference, waist to hip ratio.
1980, constata-se que a obesidade nas crianças de 6 a 11 anos aumentou em $67 \%$ entre os meninos e em $41 \%$ entre as meninas. Atualmente, $25 \%$ das crianças americanas são consideradas obesas, sendo que a maioria pertence a classes sociais com menor poder aquisitivo ${ }^{2}$. No Brasil, as crianças mais atingidas pela obesidade ainda pertencem às classes sociais mais privilegiadas, apesar da tendência recente de uma mudança nesse perfil ${ }^{3,4}$. O Instituto Nacional de Alimentação e Nutrição (INAN) aponta que a obesidade infantil no Brasil atinge $16 \%$ das crianças ${ }^{5}$. 
Apesar de não haver dúvidas quanto ao real aumento da obesidade nas crianças, persistem questões quanto ao melhor critério diagnóstico nessa faixa etária. Segundo Fisberg ${ }^{6}$, o peso por estatura em crianças e o índice de massa corporal em adolescentes seriam os melhores indicadores. O autor cita dificuldades na avaliação da composição corporal em crianças e também o desconhecimento quanto aos limites do percentual de gordura, associado a riscos em relação à saúde nessa faixa etária. Davies \& Preece ${ }^{7}$ admitem ser insatisfatório o número de técnicas disponíveis para o estudo da composição corporal de crianças e adolescentes quando comparado aos adultos. Parte do problema pode ser atribuído às profundas alterações na composição corporal que ocorrem no processo fisiológico do crescimento, principalmente quanto às porcentagens de gordura, músculos e ossos ${ }^{8}$.

Um dos problemas no diagnóstico de sobrepeso e obesidade, em populações jovens, é o desacordo quanto ao "ponto de corte" ou limites para sua identificação. Segundo Dietz \& Bellizze ${ }^{9}$, o sobrepeso na infância e adolescência é caracterizado por um índice de massa corporal por idade (IMC/idade) acima do percentil 85 e obesidade acima do percentil 95, em relação ao padrão do National Health and Nutrition Examination Survey (NHANES II-III). Para outros autores, é possível a adoção de limites do IMC, em valores absolutos, por faixas etárias, durante o período de crescimento, sem um considerável comprometimento da especificidade e sensibilidade do diagnóstico ${ }^{10}$. A adoção de padrões regionais de IMC também tem sido proposta ${ }^{11}$.

Em termos práticos, na perspectiva dos serviços de saúde, é desejável que se utilize um critério diagnóstico simples, de baixo custo, reproduzível e confiável, que tenha alta sensibilidade e especificidade, minimizando, assim, a ocorrência de diagnósticos falsos positivos ou negativos. Recentemente, Cole et al. ${ }^{12}$, baseados num pool de estudos do perfil do IMC por idade em vários países, inclusive o Brasil, propuseram limites para sobrepeso e obesidade para a faixa etária de 2 a 20 anos para uso internacional.

Este estudo se propõe a verificar o grau de concordância dessa classificação com a adiposidade de escolares préadolescentes, na faixa etária de 6 a 10 anos de idade. Para isso, foram comparados os limites propostos por Cole et al. ${ }^{12}$ com: a) adiposidade estimada a partir das dobras cutâneas tricipital, subescapular e da perna ${ }^{13}$, medidas consideradas boas indicadoras de adiposidade em crianças $^{14,15}$; b) as circunferências da cintura e do quadril e a razão cintura-quadril como indicadores de adiposidade visceral e central ${ }^{16}$.

\section{Métodos}

O estudo foi realizado em Brasília (DF), entre os anos de 2000 e 2001, envolvendo crianças pré-adolescentes, na faixa etária de 6 a 10 anos, de ambos os sexos, estudantes do Centro Educacional da Católica de Brasília (CECB). Essa instituição de ensino privado localiza-se no campus da Universidade Católica de Brasília (UCB) e atende estudantes de classe média e média alta. O estudo foi aprovado pelo comitê de ética da UCB e autorizado pelos pais dos alunos após o recebimento de uma circular explicativa. Foram incluídas na pesquisa todas as crianças na faixa etária do estudo e cujos pais autorizaram a participação.

As crianças foram examinadas no Laboratório de Avaliação Nutricional (LAN) da Universidade Católica de Brasília, como parte do projeto PREVINE (Programa de Estudos e Vigilância Nutricional de Escolares), desenvolvido pela universidade. Nesse projeto, são realizados medidas anuais de peso e estatura e cálculo do IMC dos alunos do CECB, seguidos de registro em cartão impresso, que fica de posse da família. O projeto visa ao diagnóstico precoce e orientação dos alunos e familiares quanto a sobrepeso, obesidade e baixo peso. O número atual de alunos do CECB é de cerca de 2.500 estudantes.

As crianças foram avaliadas medindo-se peso, estatura, dobras cutâneas tricipital e subescapular e perímetro da cintura e do quadril, conforme normas propostas por Lohman et. al. ${ }^{17}$. A pesagem foi realizada com as crianças descalças, vestindo short e camiseta, em uma balança digital (Indústrias Fillizola S.A. - Brasil) com capacidade de $0-150 \mathrm{~kg}$ e precisão de $100 \mathrm{~g}$. Para a coleta da estatura, as crianças foram colocadas descalças, em posição ereta, encostadas numa superfície plana vertical, braços pendentes com as mãos espalmadas sobre as coxas, os calcanhares unidos e as pontas dos pés afastadas, formando ângulo de $60^{\circ}$, joelhos em contato, cabeça ajustada ao plano de Frankfurt e em inspiração profunda. A medida foi feita em triplicata, para o cálculo do valor médio, utilizando-se um estadiômetro tipo trena (Sanny Kirchner \& Wilhelm, Medizintechnik, Germany) fixado à parede, com capacidade de $2 \mathrm{~m}$ e precisão de $0,1 \mathrm{~cm}$. Para a medida das dobras cutâneas, foi utilizado um adipômetro (Lange Beta Technology Incorporated, Cambridge, Maryland) com precisão de 0,5 mm. Tanto a dobra cutânea tricipital como a subescapular foram medidas com as crianças em posição ereta e os braços pendentes naturalmente. As duas dobras foram medidas no lado direito, em triplicata, para o cálculo da média. A dobra tricipital foi medida no ponto médio do braço entre o ponto acromial da escápula e o olécrano da ulna. A dobra subescapular foi medida num ponto localizado imediatamente abaixo do ângulo inferior da escápula direita. A dobra cutânea da panturrilha medial foi medida no ponto de maior circunferência da perna. O perímetro da cintura foi medido com fita métrica metálica ao nível do umbigo, com a criança em expiração e o perímetro do quadril na área de maior circunferência na região glútea.

As crianças foram classificadas como eutróficas, com sobrepeso ou obesidade de acordo com IMC/idade, segundo os limites propostos por Cole et al. ${ }^{12}$. Crianças abaixo do $5^{\circ}$ percentil do IMC/idade foram classificadas como de baixo peso ${ }^{18}$.

A porcentagem de gordura corporal ( $\%$ GC) foi estimada a partir da somatória das dobras tricipital e subescapular e tricipital e da panturrilha, usando as equações preditivas propostas por Slaughter et. al. ${ }^{13}$, que levam em consideração as variações sexuais e de etnia nos seus cálculos. Essas medidas tem sido utilizadas por vários autores como indicadoras de gordura corporal periférica em crianças $^{14}$. O 
perímetro da cintura, por sua vez, é considerado um bom indicador de obesidade e gordura visceral em adultos e crianças ${ }^{14,19,20}$.

A estatística descritiva compreendeu o cálculo das médias $(x)$, desvio padrão $(d p)$ e freqüência percentual (\%). O coeficiente de correlação linear de Pearson foi utilizado para avaliar o nível de correlação entre as variáveis testadas. Utilizou-se o teste $t$ de Student para amostras independentes, para comparar os resultados médios das varáveis analisadas. Foram consideradas significantes as diferenças com $p \leq 0,05$. Os dados foram analisados usando o programa SPSS 10.0 (SPSS Inc).

\section{Resultados}

Foram avaliados 528 escolares, sendo 273 do sexo feminino $(51,7 \%)$ e 255 do sexo masculino ( $48,3 \%)$. Esse número de alunos corresponde a cerca de $90 \%$ dos estudantes da instituição, na faixa etária avaliada, sendo que os demais não foram avaliados por estarem ausentes no dia da coleta das medidas ou pela falta de consentimento dos pais para participarem da pesquisa. Na Tabela 1 , encontram-se as porcentagens de crianças eutróficas, com baixo peso, sobrepeso e obesidade no grupo de escolares avaliados. O sobrepeso e a obesidade, em conjunto, atingiram $18,8 \%$ dos meninos e $21,2 \%$ das meninas.
Nas Tabelas 2 e 3, são apresentadas as médias da porcentagem de gordura corporal, da somatória das dobras cutâneas tricipital e subescapular e tricipital e da panturrilha das crianças eutróficas, com sobrepeso e obesidade. Foram excluídas da análise as crianças com baixo peso, pelo pequeno número encontrado na amostra estudada. Como pode-se verificar, há uma diferença significativa entre as crianças normais e aquelas com sobrepeso, e estas em relação às crianças obesas. Fazem exceção os meninos com obesidade em relação aos com sobrepeso, apesar da tendência para valores maiores nos obesos em relação aos com sobrepeso.

$\mathrm{Na}$ Tabela 4, estão os valores médios do perímetro da cintura nas crianças estudadas, segundo o IMC por idade. Como pode-se observar, eles são significativamente mais elevados nas crianças com sobrepeso e obesidade em relação às normais. A mesma tendência foi observada em relação ao perímetro do quadril. A razão cintura-quadril, por outro lado, não apresentou diferenças comparandose as crianças eutróficas e as com sobrepeso e obesidade, em ambos os sexos.

As Figuras 1 e 2 ilustram as correlações significativas entre o IMC e a porcentagem de gordura corporal em meninos ( $p<0,02 ; r=+0,843)$ e meninas $(p<0,02 ; r$ $=+0,774)$; os perímetros da cintura em meninos $(\mathrm{p}<$ $0,02 ; r=+0,884)$ e meninas $(p<0,02 ; r=+0,892)$; e do quadril em meninos ( $p<0,02 ; r=+0,842)$ e meninas $(p<0,02 ; r=+0,836)$.

Tabela 1 - Freqüência de escolares com baixo peso, sobrepeso e obesidade, na faixa etária de 6 a 10 anos, de acordo com o IMC/idade 12,18 - Brasília, DF

\begin{tabular}{lcccccc}
\hline Estado nutricional & \multicolumn{2}{c}{ Meninos (255) } & \multicolumn{2}{c}{ Meninas (273) } & \multicolumn{2}{c}{ Todos (528) } \\
(IMC/idade) & $\mathbf{N}$ & \% & $\mathbf{N}$ & \% & N & \% \\
\hline Eutrófico & 199 & 78,0 & 208 & 76,2 & 407 & 77,1 \\
Baixo peso & 8 & 3,1 & 7 & 2,6 & 15 & 2,8 \\
Sobrepeso & 33 & 12,9 & 44 & 16,1 & 77 & 14,6 \\
Obesidade & 15 & 5,9 & 14 & 5,1 & 29 & 5 \\
\hline
\end{tabular}

Os valores entre parênteses referem-se ao número de escolares.

Tabela 2 - Porcentagem de gordura corporal (\%gc) e somatório médio das dobras cutâneas tricipital e subescapular ( $\Sigma$ dcts) em escolares, na faixa etária de 6 a 10 anos, avaliados segundo o IMC/idade - Brasília, DF

\begin{tabular}{|c|c|c|c|}
\hline \multirow[t]{2}{*}{$\begin{array}{l}\text { Estado nutricional } \\
\text { (IMC/idade) }\end{array}$} & \multicolumn{3}{|c|}{ Adiposidade } \\
\hline & 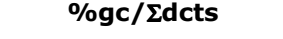 & 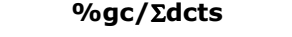 & 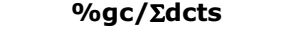 \\
\hline Eutróficas & $\begin{array}{c}16,2 \pm 4,3^{a} / 7,2 \pm 4,8^{a} \\
{[199]}\end{array}$ & $\begin{array}{c}19,0 \pm 4,0 a / 20,6 \pm 5,5^{a} \\
{[208]}\end{array}$ & $\begin{array}{c}17,7 \pm 4,4^{a} / 18,9 \pm 5,4^{a} \\
{[407]}\end{array}$ \\
\hline Sobrepeso & $\begin{array}{c}27,0 \pm 4,9^{b} / 30,3 \pm 7,1^{b} \\
{[33]}\end{array}$ & $\begin{array}{c}27,0 \pm 5,0^{b} / 32,6 \pm 7,9^{b} \\
{[44]}\end{array}$ & $\begin{array}{c}27,0 \pm 5,0^{b} / 31,6 \pm 7,6^{b} \\
{[77]}\end{array}$ \\
\hline Obesidade & $\begin{array}{c}29,2 \pm 5,5 / 33,6 \pm 7,7 \\
{[15]}\end{array}$ & $\begin{array}{c}30,8 \pm 4,0^{c} / 39,0 \pm 6,9^{c} \\
{[14]}\end{array}$ & $\begin{array}{c}30,0 \pm 4,5^{c} / 36,3 \pm 7,7^{c} \\
{[29]}\end{array}$ \\
\hline
\end{tabular}

Diferença significativa entre $a, b, c(p<0,02)$. Os valores entre colchetes referem-se ao número de escolares. 
Tabela 3 - Porcentagem de gordura corporal (\%gc) e somatório médio das dobras cutâneas tricipital e da panturrilha ( $\Sigma$ dctp) em escolares, na faixa etária de 6 a 10 anos, avaliados segundo o IMC/idade - Brasília, DF

\begin{tabular}{|c|c|c|c|}
\hline $\begin{array}{l}\text { Estado nutricional } \\
\text { (IMC/idade) }\end{array}$ & 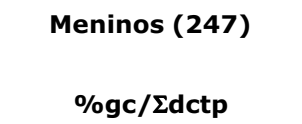 & 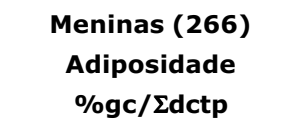 & 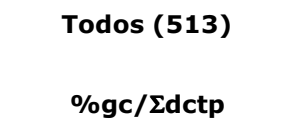 \\
\hline Eutróficas & $\begin{array}{c}18,0 \pm 4,9^{a} / 23,1 \pm 6,7^{a} \\
{[199]}\end{array}$ & $\begin{array}{c}22,4 \pm 4,6^{a} / 28,4 \pm 7,6^{a} \\
{[208]}\end{array}$ & $\begin{array}{c}20,3 \pm 5,2^{a} / 25,8 \pm 7,6^{a} \\
{[407]}\end{array}$ \\
\hline Sobrepeso & $\begin{array}{c}30,9 \pm 6,5^{b} / 40,7 \pm 8,8^{b} \\
{[33]}\end{array}$ & $\begin{array}{c}31,2 \pm 5,5^{\mathrm{b}} / 42,9 \pm 9,0^{\mathrm{b}} \\
{[44]}\end{array}$ & $\begin{array}{c}31,1 \pm 5,9^{\mathrm{b}} 41,9 \pm 8,9^{\mathrm{b}} \\
{[77]}\end{array}$ \\
\hline Obesidade & $\begin{array}{c}31,4 \pm 5,8 / 41,4 \pm 7,9 \\
{[15]}\end{array}$ & $\begin{array}{c}34,2 \pm 5,7 / 47,8 \pm 9,4^{c} \\
{[14]}\end{array}$ & $\begin{array}{c}32,8 \pm 5,8 / 44,6 \pm 9,1 \\
{[29]}\end{array}$ \\
\hline
\end{tabular}

Diferença significativa entre $a, b, c(p<0,02)$. Os valores entre colchetes referem-se ao número de escolares.

Tabela 4 - Valores médios dos perímetros da cintura, quadril $(\mathrm{cm})$ e razão cintura-quadril em escolares, na faixa etária de 6 a 10 anos, avaliados segundo o IMC/idade - Brasília, DF

\begin{tabular}{|c|c|c|c|c|c|c|}
\hline \multirow{3}{*}{$\begin{array}{l}\text { Estado nutricional } \\
\text { (IMC/idade) }\end{array}$} & \multicolumn{2}{|c|}{ Meninos (247) } & \multicolumn{2}{|c|}{ Meninas (266) } & \multicolumn{2}{|c|}{ Todos (513) } \\
\hline & \multicolumn{6}{|c|}{ Perímetro da cintura } \\
\hline & $\mathbf{N}$ & $x \pm d p$ & $\mathbf{N}$ & $x \pm d p$ & $\mathbf{N}$ & $x \pm d p$ \\
\hline Eutróficos & 199 & $57,4 \pm 4,1^{a}$ & 208 & $56,3 \pm 4,0^{a}$ & 407 & $56,9 \pm 4,1^{a}$ \\
\hline Sobrepeso & 33 & $67,8 \pm 4,4^{b}$ & 44 & $64,2 \pm 5,4^{b}$ & 77 & $65,9 \pm 5,3^{b}$ \\
\hline \multirow[t]{3}{*}{ Obesidade } & 15 & $68,7 \pm 6,6$ & 14 & $68,7 \pm 5,7 c$ & 29 & $68,3 \pm 6,3^{c}$ \\
\hline & \multicolumn{6}{|c|}{ Perímetro do quadril } \\
\hline & $\mathbf{N}$ & $x \pm d p$ & $\mathbf{N}$ & $x \pm d p$ & $\mathbf{N}$ & $x \pm d p$ \\
\hline Eutróficos & 199 & $66,5 \pm 5,2^{a}$ & 208 & $68,8 \pm 5,6^{a}$ & 407 & $67,7 \pm 5,6^{a}$ \\
\hline Sobrepeso & 33 & $77,1 \pm 6,2^{b}$ & 44 & $76,9 \pm 7,3^{b}$ & 77 & $77,0 \pm 6,8^{b}$ \\
\hline \multirow[t]{3}{*}{ Obesidade } & 15 & $79,7 \pm 6,6$ & 14 & $79,7 \pm 7,5$ & 29 & $79,3 \pm 7,1$ \\
\hline & \multicolumn{6}{|c|}{ Razão cintura-quadril } \\
\hline & $\mathbf{N}$ & $x \pm d p$ & $\mathbf{N}$ & $x \pm d p$ & $\mathbf{N}$ & $x \pm d p$ \\
\hline Eutróficos & 199 & $0,87 \pm 0,02$ & 208 & $0,82 \pm 0,05$ & 407 & $0,84 \pm 0,05$ \\
\hline Sobrepeso & 33 & $0,88 \pm 0,06$ & 44 & $0,84 \pm 0,04$ & 77 & $0,86 \pm 0,05$ \\
\hline Obesidade & 15 & $0,86 \pm 0,04$ & 14 & $0,86 \pm 0,03$ & 29 & $0,86 \pm 0,03$ \\
\hline
\end{tabular}

Diferença significativa entre $a, b, c(p \leq 0,02)$.

\section{Discussão}

A freqüência de sobrepeso e obesidade encontrada nos escolares avaliados é elevada e semelhante ao observado no sudeste do Brasil ${ }^{21}$, confirmando a gravidade do problema em nossos escolares.

O uso do IMC por idade, de acordo com os limites propostos por Cole et al. ${ }^{12}$, mostrou boa concordância com a adiposidade crescente nos grupos. Este resultado corrobora as observações de outros autores sobre a boa correlação entre a adiposidade e o IMC em crianças $^{22}$.

Tanto o perímetro da cintura como o do quadril mostraram valores médios significativamente diferentes nas crianças com sobrepeso e obesidade, quando comparadas às crianças normais, ao contrário da razão cinturaquadril que foi semelhante nos grupos estudados. Esta observação confirma os achados de outros autores, mostrando que a razão cintura-quadril não é um bom indicador de obesidade visceral no período pré-puberal ${ }^{23}$.

Correlações significativas foram notadas entre o IMC e a gordura corporal e o IMC e os perímetros da cintura e do quadril. Esses achados estão de pleno acordo com outras observações na literatura no que se refere à gordura corporal, porém, são poucas as citações em relação ao perímetro da cintura e do quadril 24 . 


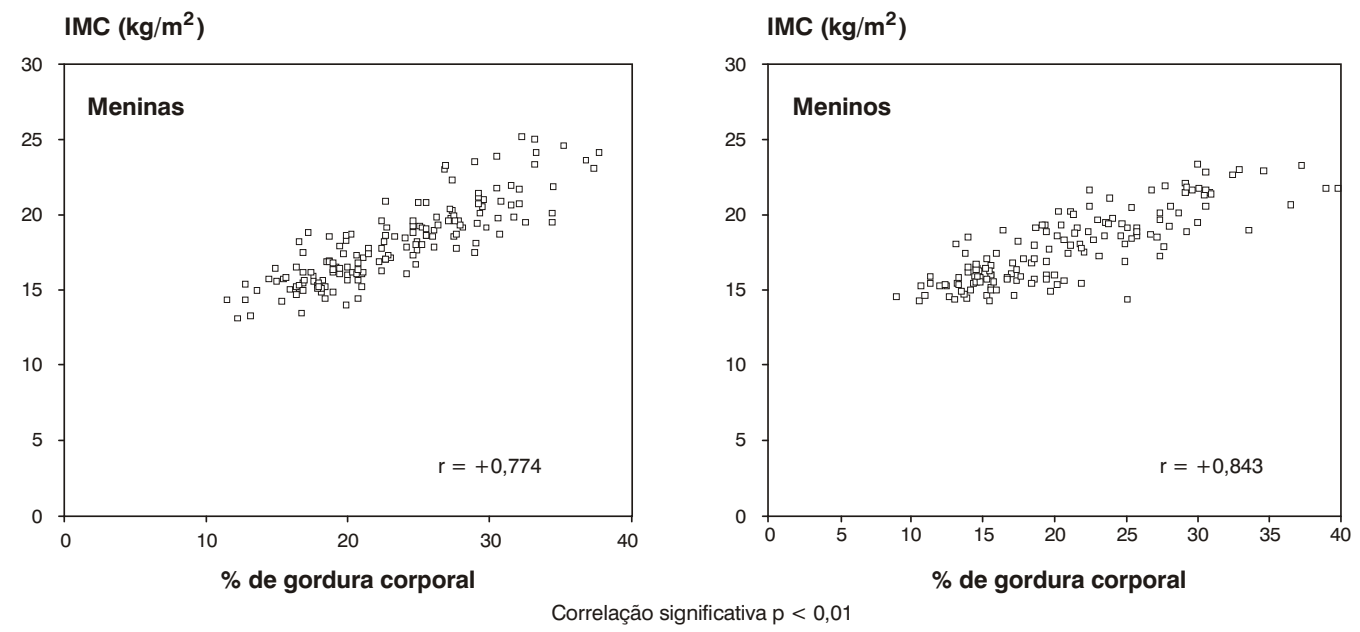

Figura 1 - Gráfico de dispersão entre o índice de massa corporal e a porcentagem de gordura corporal em escolares, na faixa etária de 6 a 10 anos

IMC $\left(\mathbf{k g} / \mathrm{m}^{2}\right)$

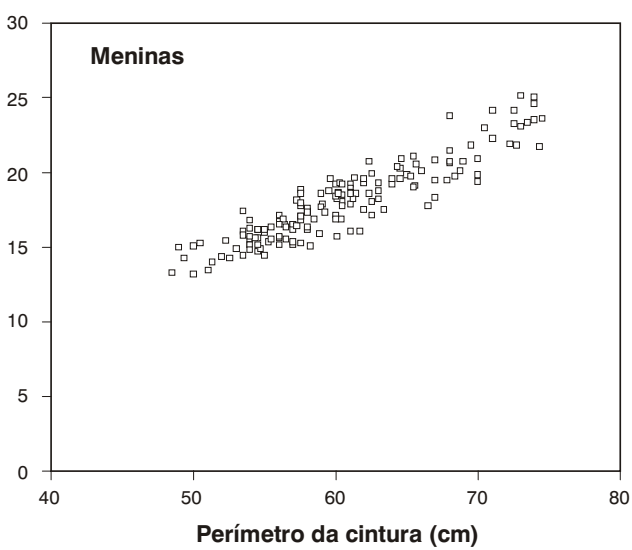

Correlação significativa $p<0,01$

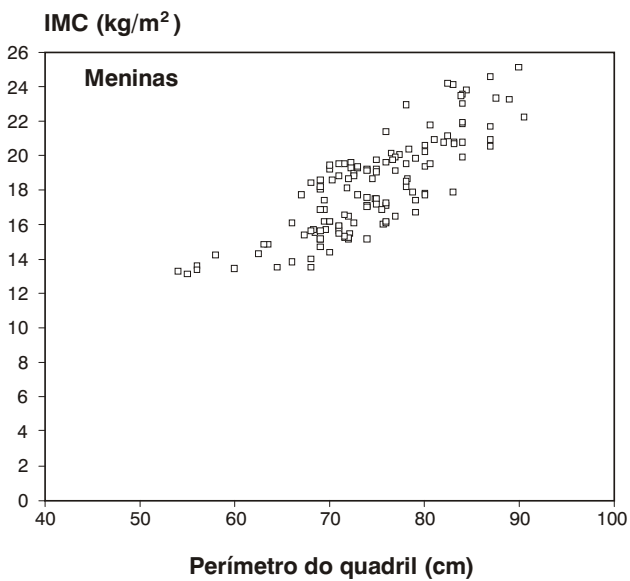

Correlação significativa $p<0,01$
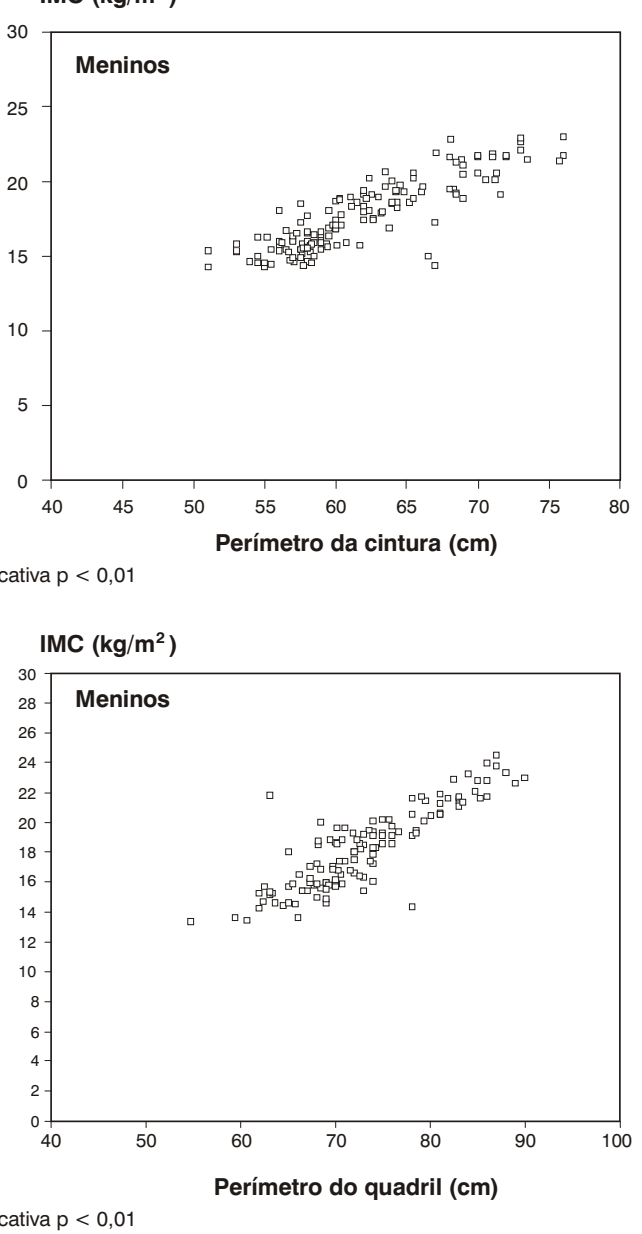

Figura 2 - Gráfico de dispersão entre o índice de massa corporal e os perímetros da cintura e do quadril em escolares, na faixa etária de 6 a 10 anos 
A validade do uso do IMC como indicador de adiposidade em crianças vem sendo demonstrada em vários estudos 22,25, porém, os limites ou "pontos de corte" do IMC de aplicação internacional, para o diagnóstico de sobrepeso e obesidade em crianças, têm sido contestados devido a variações raciais, segundo alguns autores 26,27 . No nosso meio, considerando o aspecto multirracial do povo brasileiro, os limites propostos parecem ter boa concordância com a adiposidade, pelo menos em escolares. No presente estudo, apesar da utilização de um método duplamente indireto para a estimativa da gordura corporal, os valores encontrados apresentaram uma associação significativa com a classificação proposta, baseada no IMC por idade.

$O$ resultados deste estudo mostram que é adequada a escolha do IMC/idade, baseado em padrão internacional, como indicador diagnóstico de sobrepeso e obesidade em escolares. Ele apresentou concordância com o excesso de adiposidade corpórea, calculada a partir da medida de dobras cutâneas, e com as gorduras visceral e central, estimadas indiretamente a partir das circunferências de cintura e quadril. A adoção de critérios uniformes e simples de serem utilizados, como é o caso do IMC/idade, facilitaria a comparação de resultados entre regiões e países, assim como a avaliação do impacto de medidas preventivas e curativas relativas ao problema. Considerando que o estudo foi limitado quanto a faixa etária e etnia, além do uso de métodos que não são considerados de referência para o cálculo da adiposidade, fazem-se necessários mais estudos, para que esse indicador antropométrico possa ser adotado de maneira sistemática em nosso meio.

\section{Agradecimentos}

A Vânia Pereira Nunes, pela dedicação e trabalhos técnicos. Ao Prof. Eduardo da Silva Sena, coordenador do SEDEFE - Setor de Educação Física e Esportes do Centro Educacional da Católica de Brasília, pelo apoio e incentivo.

\section{Referências}

1. World Health Organization. Obesity. Preventing and managing the global epidemic: Report of WHO consultation group on obesity. Geneva: WHO; 1997.

2. Dietz WH. Prevention of childhood obesity. Pediatr Clin North Am. 1986;33:823-33.

3. Cyrino ES, Nardo N. Subsídios para prevenção e controle da obesidade. Rev Bras Ativ Física e Saúde. 1996;1:15-25.

4. Monteiro CA, Conde WL. Tendência secular da obesidade segundo estratos sociais: Nordeste e Sudeste do Brasil. Arq Bras Endocrinol Metab. 1999;43:186-94.

5. Brasil. Ministério da Saúde, Instituto Nacional de Alimentação e Nutrição. Pesquisa Nacional sobre Saúde e Nutrição. Perfil de crescimento da população brasileira de 0-25 anos. Brasília: INAN; 1990.

6. Fisberg M. Obesidade na infância e adolescência. São Paulo (SP): Fundação BYK; 1995.
7. Davies PSW, Preece MA. Body composition in children: methods of assessment. In: Tanner JM, Preece MA, editors. The physiology of human growth. Cambridge: Cambridge University Press; 1989. p. 95-107.

8. Malina RM, Bouchard C. Growth maturation and physical activity. Champagnat (II): Human Kinectics Books; 1991.

9. Dietz WH, Bellizzi MC. Introduction: the use of body mass index to assess obesity in children. Am J Clin Nutr. 1999;70(1):1235-55.

10. Monteiro POA, Victora CG, Barros FC, Tomasi E. Diagnóstico de sobrepeso em adolescentes: estudo de diferentes critérios para o índice de massa corporal. Rev Saude Publica. 1999;34:506-13.

11. Anjos LA, Veiga, GV, Castro IRR. Distribuição dos valores do índice de massa corporal da população brasileira até 20 anos. Rev Panamer Saude Publica. 1998;3:42-50.

12. Cole TJ, Bellizzi MC, Flegal KM, Dietz WH. Establishing a standard definition for child overweight and obesity worldwide: international survey. BMJ. 2000;320:1240-3.

13. Slaughter MH, Lohman TG, Boileau RA, Horswill CA, Stillman RJ, VanLoan $M$, et al. Influence of maturation on relationship of skinfold to body density: a cross-sectional study. Human Biology. 1988;56:681-9.

14. Sigulem DM, Devincenzi MV, Lessa AC. Diagnóstico do estado nutricional da criança e do adolescente. J Pediatr (Rio J). 2000;76 Supl 3:275-84

15. Heyward VH, Stolarczyk LM. Applied body composition assessment. Champagnat (II): Human Kinectics Books; 1996.

16. McCarthy HD, Ellis SM, Cole TJ. Central overweight and obesity in British youth aged 11-16 years: cross sectional survey of waist circumference. BMJ. 2003;326:624-6.

17. Lohman TG, Roche AFE, Martorell R. Anthropometric standardization reference manual assessment. Champagnat (II): Human Kinectics Books; 1988.

18. Hammer LD, Kraemer LD, Wilson DM, Ritter PL, Dornbusch SM. Standardized percentile curves of body-mass index for children and adolescents. Am J Dis Child. 1991;145:259-63.

19. Maffeis C, Grezzani A, Pietrobelli A, Provera S, Tato LL. Does waist circumference predict fat gain in children? Int J Obes Relat Metab Disord. 2001;25:978-83.

20. Sarria A, Moreno LA, Garcia-Llop LA, Fleta J, Morellon MP, Bueno $M$. Body mass index, triceps skinfold and waist circumference in screening for adiposity in male children and adolescents. Acta Paediatr. 2001;90:387-92.

21. Neutzling MB, Taddei JA, Rodrigues, EM, Sigulem DM. Overweight and obesity in Brazilian adolescents. Int J Obes Relat Metab Disord. 2000;24:869-74.

22. Pietrobelli A, Faith MS, Allison DB, Gallagher D, Chiumello G, Heymsfield SB. Body mass index as a measure of adiposity among children and adolescents: a validation study. J Pediatr. 1998;132:204-10.

23. World Health Organization. Physical status: the use and interpretation of anthropometry. Technical Report Series 854. Geneva: WHO; 1995.

24. Higgins PB, Gower BA, Hunter GR, Goran ML. Defining healthrelated obesity in prepubertal children. Obes Res. 2001;9:233-40.

25. Mei Z, Grummer-Strawn LM, Pietrobelli A, Goulding A, Goran MI, Dietz WH. Validity of body mass index compared with other body-composition screening indexes for the assessment of body fatness in children and adolescent. Am J Clin Nutr. 2002;75:978-85.

26. Deurenberg P, Deurenberg-Yap M. Differences in body composition assumptions across ethnic groups: practical consequences. Curr Opin Clin Nutr Metab Care. 2001;4:377-83.

27. Choo V. WHO reassesses appropriate body-mass index for Asian populations. Lancet. 2002;360:235.

\section{Correspondência:}

Rodolfo Giugliano

Universidade Católica de Brasília - UCB

Laboratório de Avaliação Nutricional - LAN

Prédio São Gaspar Bertoni [bloco M] - sala 121

QS 07 Lote 1 Águas Claras

CEP 71966700 - Taguatinga, DF

Tel.: (61) 356.9338 - Fax: (61) 356.3010 